Article

\title{
A Robust Timetabling Model for a Metro Line with Passenger Activity Information
}

\author{
Lai Wei * and Zhenzhou Yuan \\ MOE (Ministry of Education) Key Laboratory for Urban Transportation Complex Systems Theory and \\ Technology, Beijing Jiaotong University, Beijing 100044, China; zzyuan@bjtu.edu.cn \\ * Correspondence: 15114222@bjtu.edu.cn; Tel.: +86-010-5168-8534
}

Received: 22 July 2017; Accepted: 21 August 2017; Published: 25 August 2017

\begin{abstract}
Timetable design is crucial to the reliability of a metro service. In terms of the delays caused by passengers' boarding and alighting behaviors during rush hours, the planned timetable for a metro line with high-frequency service tends to be difficult to implement. General oversaturation events, rather than accidents or track damage, still have a significant impact on metro systems, so that trains are canceled and delayed. When the activity reality diverges from the real-time or historical information, it is imperative that dispatchers present a good solution during the planning stage in order to minimize the nuisance for passengers and reduce the crowding risk. This paper presents a robust timetabling model (RTM) for a metro line with passenger activity information, which takes into account congestion and buffer time adjustments. The main objective pursued by dispatchers in the model is the enhancement of punctuality while minimizing train delays by adjusting the buffer time. By explicitly taking the passenger activity information into account, a mixed integer nonlinear programming (MINLP) model was developed, and a genetic algorithm (GA) is proposed to solve the model. Finally, numerical experiments based on the Batong line of the Beijing Metro were carried out, the results of which verify the effectiveness and efficiency of our method.
\end{abstract}

Keywords: robust; timetabling; metro; passenger activity information

\section{Introduction}

With the increasing size and range of urban networks, the metro, as an important component of public transportation (PT), makes a significant contribution to the daily service in many cities. A considerable factor for retaining existing passengers and attracting new users is improving the reliability of the system by designing a feasibly robust timetable during the planning stage. Thus, guaranteeing a pre-determined timetable for a more robust metro line has become a key task. Owing to its more intelligent control systems and information sharing technology, rail-based metro systems, such as Shanghai's urban rail transit (URT), Hong Kong's mass transit railway (MTR), and the Tokyo Metro, are particularly important to a metropolis. The operation of trains is characterized by high-frequency service in several metro systems, where the minimum headway between two successive trains is usually two to five minutes. Especially on the Yizhuang line of the Beijing Metro, it may be as little as $90 \mathrm{~s}$ with the development of advanced signaling systems [1]. In big cities, metro systems transport millions of passengers every day. However, congestion during the morning and evening peak times usually has an adverse effect on the scheduled departure from every station depending on the planning timetable. In other words, there are many real situations that make the original solution virtually unfeasible, such as metro doors or platform screen doors not closing due to crowding passengers. These potential events might exacerbate delays and lead to unreliability of railway systems, which includes train delays, insufficient loading capacity, broken synchronizations, and canceled trains, and may prevent an area from obtaining metro service. Thus, the key issue for 
enhancing the robustness of a timetable is determining how to estimate the interactions between the trains and passengers from the information collected.

In train timetabling, the word "disturbance" is a common term for these disruptions and is formally defined as the mistakes, malfunctions, or deviating conditions that occur in the railway system or its environment and influence the railway traffic [2]. Lacking detailed passenger activity information, previous studies on timetable scheduling were conservatively addressed within idealized transit circumstances [3-5].

Passenger activity information is defined in this paper as information available to the transit system operator, and concerns the numbers of passengers boarding and disembarking from trains, and waiting on the platform. The most important input data in operation planning studies is the number of passengers boarded and alighted, determined by the aforementioned IC systems. In the planning stage, based on these input data, the scheduling running time and dwell time are computed for different weekdays and holidays by adjusting the buffer time. In practice, many unscheduled events may occur, and the estimated buffer time may change. Therefore, in real-world conditions, a robust timetable is required.

Dwell time refers to the time a train spends at a scheduled stop without moving. Typically, this time is spent on boarding or alighting passengers according to the planned schedule. Dwell time is one common measure of efficiency in PT, with appropriate determination of dwell times being universally desirable.

Delays of trains occur since planned schedule are not able to handle real-time passenger activities, which is usually different from the historical data. However, the planned timetable is a fixed and deterministic plan. In order to cope with unscheduled events, a timetable therefore usually contains time supplements between consecutive train movements on the same part of the infrastructure. By the time supplements, part of the delays can be absorbed without giving rise to delays. The above-described time supplement is defined as buffer time in this study.

The rescheduling process by the means of RTM deals with unexpected events occurring in the original plan. Generally, the quality of the rescheduled timetable is measured by the difference between the two timetables before and after implementation of the proposed approach. That is, the rescheduled timetable is better if it alters the original timetable less. Therefore, from the dispatchers' perspective, the reliability of a predetermined timetable is a key component in achieving full integration of the operational plan.

One of essential tasks for creating a reliably predetermined timetable is setting the optimal buffer time. Naturally, buffer time needs to be added by considering unscheduled performances which are derived from an imbalance between supply and demand, such as overloaded and almost empty trains, when formulating a timetable. Typically, the correct amount of buffer time finds an optimal balance between extra waiting time, having a superfluous number of trains, and insufficient robustness. The amount of buffer time can greatly diminish the level of service (LOS) by causing an inconvenience to passengers. Conversely, if the buffer time is insufficient, train drivers are not likely able to catch up to the planned timetable when they fall behind, thus decreasing the service reliability. Therefore, determining the optimal buffer time that contributes to the robustness involves a tradeoff between the service quality and the operation reliability.

Various studies have been undertaken to assess the buffer time [6-8]. However, the main drawback of the optimizing buffer time approaches is the lack of ability to be applied to metro service combined with passenger activity information, whether for airlines, high-speed railways (HSR), or buses. As proven in [9], these adjustments in slack or buffer time inserted into the timetable lead to scheduling stability. This also gives drivers the incentive to catch up to the schedule since most transit agencies penalize them for being excessively late.

To improve the robustness of a timetable for a metro line, this paper seeks to minimize deviation from the planning timetable by adjusting the buffer time of the train running process so that the timetable is successfully achieved. Within the operating technology constraints of considering train 
capacity and investigating historical passengers' information, the actual dwell time was chosen by the interactional relationship between waiting passengers and trains. This approach is applied to a metro line including upstream or downstream directions of the Batong line belonging to the Beijing Metro. This is a line of 13 stations located in a suburb of Beijing. Involving deviations at the planning stage of a metro timetable may yield much better timetabling performance. Furthermore, a well-designed timetable considering punctuality of running time between two major stations can satisfy the needs of critical sections using the time control point strategy.

\subsection{Literature Review}

Metro operational management is typically classified into three levels: strategic, tactical, and operational [10]. For the metro service in a PT system, an applicable timetable is at the core of daily operations and solutions to a variety of potential issues. Based on the infrastructure construction in strategic-level decisions, such as rail line design, number and locations of stations [11], the rail transit command center (TCC) determines the stop patterns from demand estimates obtained from data gathered from IC, which can be considered a tactical-level problem. The tactical decision for selecting a stop pattern is likely to contain numerous conflicts between trains, infeasibilities in train times, headways, dwell times, and other problems. Therefore, after a tactical decision is made, the TCC can adjust and revise the preliminary plan to eliminate all conflicts by analyzing performance of the train type and timetabling [12,13].

Fundamentally, there are an enormous number of daily PT passenger trips. There are 11 million daily trips on the Tokyo Subway, nine million on the Shanghai Metro, and five million on the London Underground [13]. Delays usually occur due to the congestion caused by train doors and platform screen doors not locking when the boarding and alighting passengers are crowded into these areas. The level of congestion and assistant push on the Tokyo Subway during rush hours in the news is shown in Figure 1.

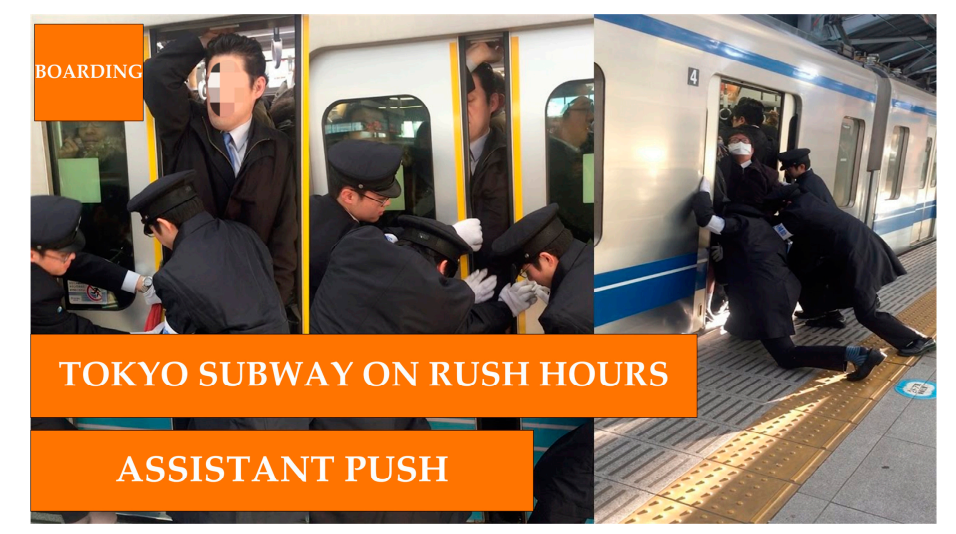

Figure 1. Common congestion and assistant push on the Tokyo Subway on rush hours.

The problem of creating a robust train timetable has received considerable attention in the literature. For instance, analytical aspects of dispatching policies were investigated for single mode of transportation (bus, train, airline, etc.) routes [3]. Osuna and Newell formulated the control strategies for an idealized PT system with the objective of minimizing the average wait per passenger [4]. Ideal buffer times are calculated on the delay distributions of the arriving trains, weighting the different types of waiting times. Moreover, standard linear programming is used at improving the Belgian railway timetable with a $40 \%$ cost decrease [6,7].

$A$ branch and bound $(B \& B)$ algorithm was proven to be technically feasible for solving nonlinear integer programming (NIP) problems characterized by large-scale calculations [2,8,14]. A B\&B algorithm was reasonably applied to railway rescheduling problems to speed up the computation 
to ensure the efficiency of real-time control. A new conflict-free timetable with feasible arrival and departure times was updated, with the objective of minimizing deviations from the original [8]. The B\&B algorithm gained further development during a single-track train timetabling study, in which three approaches were adapted to effectively reduce the solution space. First, a Lagrangian relaxation-based lower bound rule was used to dualize segments and station entering headway capacity constraints. Second, an exact lower bound rule was used to estimate the smallest train delay for resolving the remaining crossing conflicts in a partial schedule. Third, a beam search heuristic method established the tight upper bound [14]. A B\&B algorithm, along with a new heuristic beam search algorithm, is presented to solve robust train timetabling problems. The required buffer times were measured to seek the tradeoffs between the optimality, robustness, and the capacity consumption [2]. The objective of the model was developed based on the approach of Reference [15]. Bertsimas and Sim introduced a different robust approach in which the robust counterpart is of the same class and size as the nominal problem. This approach has the advantage of having the ability to control the degree of reliability under uncertainty [15].

A real time scheduling optimization of a train service in a metro rail terminal was proposed with two objectives performed in two steps. The first step investigated the problem of routing and sequencing trains with a goal of punctuality, which was solved with a fast heuristic. Scheduling train departures with the objective of optimizing the regularity of train service, under the constraint that what was achieved in the first objective does not deteriorate at the second step [16]. A scenario-based rolling horizon solution approach was proposed to dispatch a single-track train under a dynamic and stochastic environment. The study reduced the expected additional delays under different forecasted scenarios [17]. In this study, there was a conflict on balancing minimizing train delays and missed connections, in order to provide a set of feasible non-dominated schedules to support this decision process. These objectives involve making decisions about which connections to keep or drop in order to reach a compromise, because whenever train delay reduction requires cancellation of some connected services, it causes longer wait times for transferring passengers [18].

Disruptions, such as accidents or track damages, were also analyzed given their significant impact on railway operation. Given a disrupted infrastructure situation and a forecast of the characteristics of the disruption, the goal of the model was to design a disposition timetable, specifying which trains needed to still be in operation during the disruption and determining the timetable of these trains [19]. The definition and distinguishing characteristics between "disruption" and "disturbance" were demonstrated in a recent overview [20] in detail. These disruptions are less frequent than the unscheduled events caused by congestion in daily operations. In our work, the influence of unscheduled events on daily operations are taken into account rather than the abrupt disruptions in a relatively few cases.

In terms of the service reliability of PT systems, there are five types of vehicle positional situations with reference to a transfer point: Considerably ahead of schedule, ahead of schedule, on schedule, behind schedule, and considerably behind schedule. The developed optimization framework results in selected operational tactics to attain the maximum number of direct transfers, without waiting, and minimizes the total passenger travel time [21]. With the similar concept of buffer time, a balanced train timetable on a single-line railway with optimized velocity was obtained by using a combination of travel-advance strategy and a genetic algorithm (GA) [22]. Another effective way to achieve this is to use a stop-skipping pattern after the disruption. The skip-stop pattern means that a service skips some stations [23-25] as a robust strategy to insulate against uncertainty.

An inconvenience-minimized rescheduling model was proposed, based on Mixed Integer Programming (MIP) formulation, when train traffic is disrupted. Typically, the dwell times were estimated to match what would be considered a satisfactory connection at a station for extra passengers. As countermeasures against the disruption, changes of train types and rolling stock operation schedules at terminals, as well as changes in the departing order of trains and assignment of trains to a track in a station, are performed [26]. A rescheduling approach was addressed for a metro line after a disruption, 
in which the time-dependent congested demand distributions within train capacity, was taken into account. A mixed integer linear programming model (MILP) to investigate this problem was developed and it was solved with a heuristic iterative algorithm. Numerical experiments based on the data from the Beijing Yizhuang metro line were analyzed. Under different conditions, such as increasing and decreasing passenger arrival rates, and short and long disruption periods, the effectiveness and efficiency of our rescheduling method were verified [27].

\subsection{Contribution of the Paper}

Note that the concept of robust timetabling in this paper is different from the traditional concept. Our work aims to minimize the deviation from the original timetable caused by passenger congestion. To the authors' knowledge, there is limited literature on RTM for a metro line during the planning stage, in which the passenger activity information is quantified and analyzed.

The contributions made in this paper are summarized as follows. Firstly, this paper establishes a RTM for a metro line with passenger activity information, which takes into account congestion and buffer time adjustment. By determining the available buffer time at each specified station, the robustness of a metro timetable has the advantage of quicker recovery. Secondly, we developed a MINLP model for the robustness problem pursuing the least deviation by adjusting the buffer time of the train running process to achieve it. Thirdly, we analyzed the mathematical properties of our proposed model and GA to solve the RTM that was designed. Lastly, our proposed RTM is not only capable of improving the robustness for one specified station, but could also be used to improve the punctuality of the running time between two major stations by using a time control point strategy.

\subsection{Outline}

The rest of this paper is organized as follows. Section 2 establishes the essential assumptions and defines the core mechanism of our proposed model, in which the recursive relations between scheduled time and actual time in each stage of departure are obtained. The robust approach is applied in Section 3. Section 4 describes the solution algorithm, and Section 5 provides examples to illustrate the effectiveness of our proposed model and GA. Finally, Section 6 is an important extension of the model, and Section 7 is the conclusion with suggestions for future studies.

\section{Mathematical Formulation Model}

In this section, the basic components of a RTM for a metro line are presented, and then the RTM is formulated. We make a detailed description of the RTM. The metro line is represented by a set of segments and a set of stations. The stations along the line in our case refer to the places where passengers' boarding/alighting and waiting activities occur.

\subsection{Basic Rules and Assumptions}

To facilitate the presentation of the essential ideas in this paper, some rules and assumptions are made as follows:

(1) A metro line, including the length of the line, the location, and scale of the stations belonging to the line, is given as an input, and the study period is designated, such as rush hours or holidays and festivals. It is relatively simple to clarify the under-saturated scenarios in the off-peak hours if complex issues on the oversaturated conditions in the peak hours are justified clearly in our mathematical formulations.

(2) It is assumed that in the RTM of our study, detailed information about rolling stocks and signal interlocking equipment is neglected, which indicates that we considered the robust timetable designing at the operational management level.

(3) No extra trains are added, and no train is cancelled. That is, the robust re-timetabling is only for trains already in the metro system affected by the unscheduled events. 
(4) The scheduled headway for a line metro is given. It is a practical implementation in which constant headway is inputted during the given period as a way to assist frequent passengers [28]. The trains depart from the terminal on time. Therefore, the departure time of each trip from the terminal of the line is a multiple of the scheduled headway plus the first trip departure time.

(5) At a station, all passengers boarding a train obey the First-In-First-Out (FIFO) principle. This assumption is valid for PT stations, certainly including the metro. Generally, the passengers arriving early have more of a chance to board the coming train at rush hours than later ones. In order to facilitate problem formulation, this study assumes that all the queuing passengers follow the FIFO principle to board a train, which is a queuing discipline used to calibrate the excess demand based on the passenger activity information.

(6) The total buffer time allocated to each line does not exceed a required value, which does not significantly change the half-cycle time. Hence, extra operational costs caused by adding buffer time into timetable are ignored.

(7) The average number of passengers, which includes boarding passengers, alighting passengers, and through passengers, for each trip is given.

(8) The pure running time, including extra acceleration and deceleration time, between two consecutive stations is obtained by a constant speed, and is independent of the standard stop pattern used. In this case, overtaking is prohibited.

\subsection{Timetable Constraints}

This paper considers a metro line set $N=(J, E)$, where the terminus and stations in the line are numbered in increasing order. Defining the station set $J=\{1,2, \ldots, j-1, j, \ldots, n\}$, in which $n$ denotes the total number of stations (terminus not included). Let $E=\{e(j-1, j) \mid j \in J\}$ denote the train's running segment set, where the subset $e(j-1, j) \in E$ describes the segment from station $j-1$ to the subsequent adjacent station $j$. Geographically, the corresponding length of this segment $e(j-1, j)$ of one direction is $l(j-1, j)$.

During the scheduling period, the train set of the metro service is $I=\{1,2, \ldots, I-1, i, \ldots, m\}$, where $m$ is the last train for a given time period $\left[t_{0}, t_{e}\right]$. The service number of a train is increased with $i$ when it departs from the terminal. The train operation underlying our model is a high passenger demand one-way loop transit corridor operated by a single high-frequency service consisting of $n$ homogeneous stations (see Figure 2), with a limited capacity denoted by $C$.

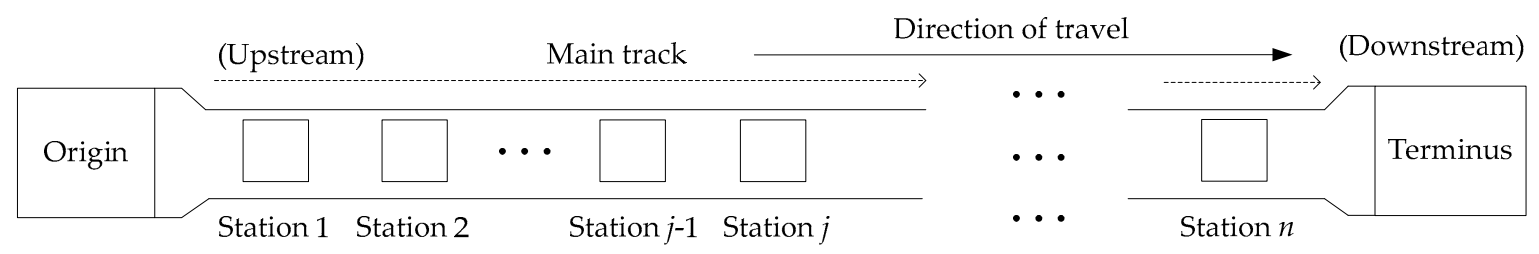

Figure 2. Train operation illustration of a metro line.

Considering all trains have the same speed between two consecutive stations and the same dwell time at each station, the safety interval $h$ can be determined in Equation (1).

$$
t_{i, j}^{\mathrm{sd}}-t_{i-1, j}^{\mathrm{sd}} \geq h \forall i \geq 2
$$

Similarly, there are the scheduled departure times $t_{i, j}^{\mathrm{sd}}$ and $t_{i-1, j}^{\mathrm{sd}}$ of trains $i$ and $i-1$ from station $j$, respectively. 
Given scheduled dwell time $\tau_{i, j}^{\mathrm{s}}$ and scheduled running time $t_{i, j-1, j}^{\mathrm{s}}$ at segment $e(j-1, j)$, the arrival $t_{i, j}^{\text {sa }}$ of train $i$ at station $j$ and departure times should satisfy Equations (2) and (3) for each train. Then, Equation (4) describes the process of train's running, divided into three phases.

$$
\begin{gathered}
t_{i, j}^{\mathrm{sd}}=t_{i, j}^{\mathrm{sa}}+\tau_{i, j}^{\mathrm{s}} \forall i, j \\
t_{i, j}^{\mathrm{sa}}=t_{i, j-1}^{\mathrm{sd}}+t_{i, j-1, j}^{\mathrm{s}} \forall i, j \geq 2 \\
t_{i, j-1, j}^{\mathrm{s}}=t_{i, j-1, j}^{\mathrm{acc}}+t_{i, j-1, j}^{\mathrm{hold}}+t_{i, j-1, j}^{\mathrm{dec}} \forall i, j \geq 2
\end{gathered}
$$

where $t_{i, j-1, j}^{\mathrm{s}}$ represents the scheduled running time of train $i$ at segment $e(j-1, j)$. According to assumption (8), the running time of the acceleration phase, the speed holding phase, and the deceleration can be calculated as:

$$
\begin{gathered}
t_{i, j-1, j}^{\mathrm{acc}}=v_{i, j-1, j} / 2 a_{i, j-1, j}^{\mathrm{acc}} \forall i, j \geq 2 \\
t_{i, j-1, j}^{\mathrm{hold}}=l(j-1, j) / v_{i, j-1, j} \forall i, j \geq 2 \\
t_{i, j-1, j}^{\mathrm{dec}}=v_{i, j-1, j} / 2 a_{i, j-1, j}^{\mathrm{dec}} \forall i, j \geq 2
\end{gathered}
$$

where $v_{i, j-1, j}$ is the speed of the speed holding phase, $a_{i, j-1, j}^{\mathrm{acc}}$ and $a_{i, j-1, j}^{\mathrm{dec}}$ are the acceleration and deceleration, respectively. Then, combining Equation (4) with Equations (5)-(7) can be rewritten as:

$$
t_{i, j-1, j}^{\mathrm{s}}=\frac{v_{i, j-1, j}}{2 a_{i, j-1, j}^{\mathrm{acc}}}+\frac{l(j-1, j)}{v_{i, j-1, j}}+\frac{v_{i, j-1, j}}{2 a_{i, j-1, j}^{\mathrm{dec}}} \forall i, j \geq 2
$$

To fulfill the metro service requirements, there are minimum and maximum speeds of trains $i$ at segment $e(j-1, j)$, denoted by $v_{i, j-1, j}^{\min }$ and $v_{i, j-1, j}^{\max }$, respectively. Thus, for each $i \in I$, the constraint is:

$$
v_{i, j-1, j} \in\left[v_{i, j-1, j}^{\min }, v_{i, j-1, j}^{\max }\right]
$$

With the minimum scheduled dwell time of train $i$ at station $j$ defined as $\tau_{i, j, \sin }^{\mathrm{s}}$, we can capture one important constraint (10). The scheduled departure time $t_{i, j}^{\text {sd }}$ of train $i$ at station $j$ should be longer than sum of its scheduled arrival time $t_{i, j}^{\mathrm{sa}}$ plus $\tau_{i, j, \mathrm{~min}}^{\mathrm{s}}$.

$$
t_{i, j}^{\mathrm{sd}} \geq t_{i, j}^{\mathrm{sa}}+\tau_{i, j, \min }^{\mathrm{s}}
$$

\subsection{Passengers' Activity Information}

To analyze the interaction between passenger distribution and headway on a metro line, we first estimated how the metro service frequency (reciprocal of the headway) is designed to allow the operator to react dynamically to unscheduled events. Figure 3 shows the demand variations of Line One (Figure 3a) and the Batong Line (Figure 3b) of the Beijing Metro in China on 27 May 2016, which was a typical workday. It is obvious that the normal workday entails a full day of operations. Certainly, in order to handle the passengers' activity information issues, special attention must be paid to daily operations. In this case, the metro service provides the static timetabling strategy resulting in a fixed headway. However, it is possible that the unbalanced demand distributions tend to cause unscheduled events, which are a focus of, and solved by, our proposed method.

The interaction between the variables used for describing the passengers' information is illustrated in Figure 4. As shown in Figure 4, the number of waiting passengers $W_{i, j}$ for train $i$ at station $j$ is equal 
to the sum of the number of waiting passengers $W_{i, j, k}$ with destination $k$ for all $k \in\{j+1, j+2, \ldots, n\}$, which is expressed as:

$$
W_{i, j}=\sum_{k=j+1}^{n} W_{i, j, k} \forall i, j
$$

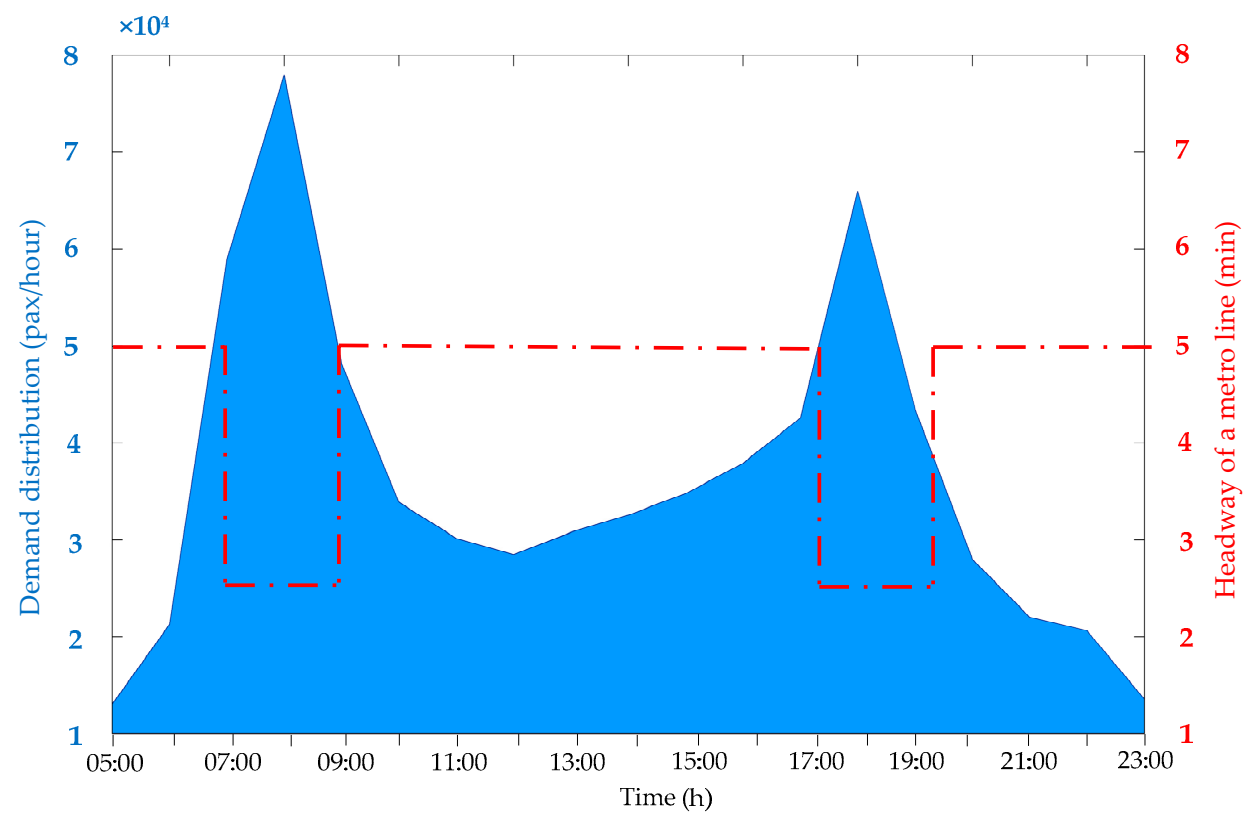

(a) Line One

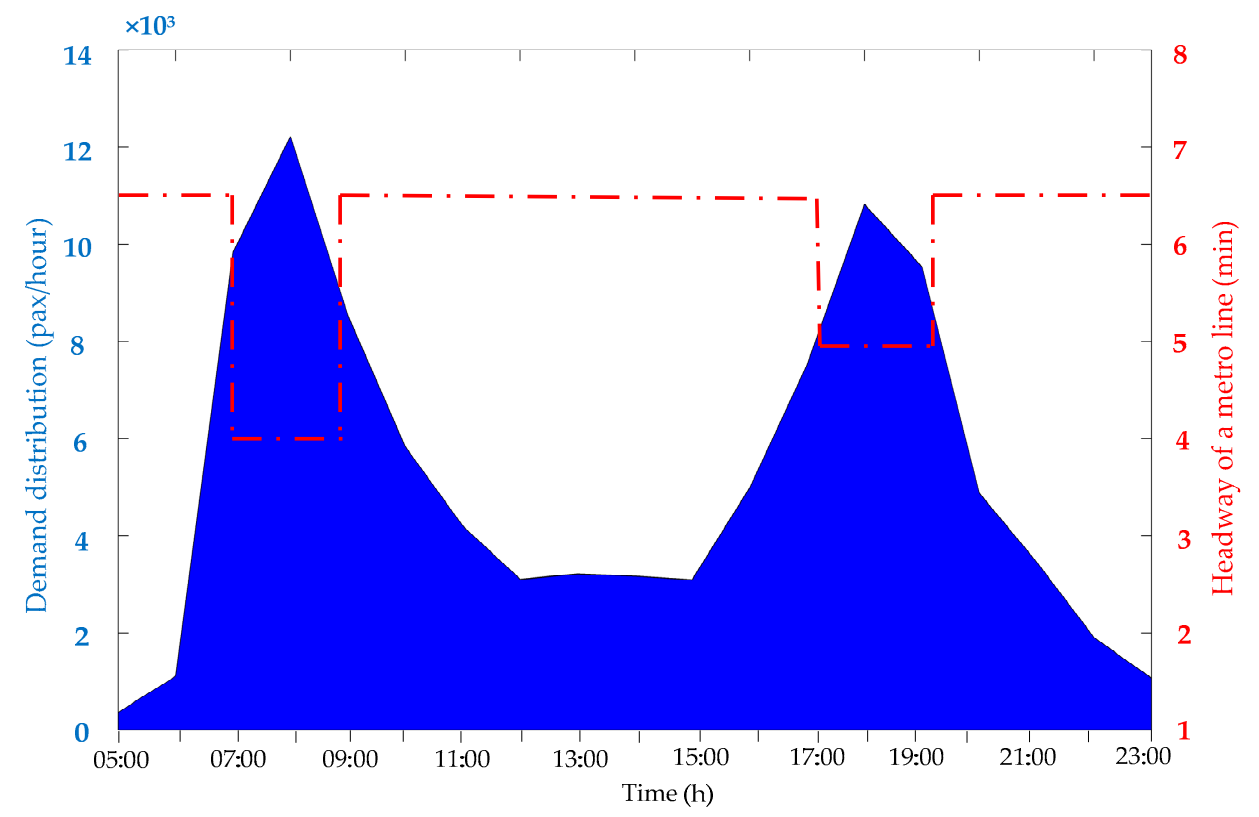

(b) Batong Line

Figure 3. Interaction between passenger distribution and headway on two metro lines. 


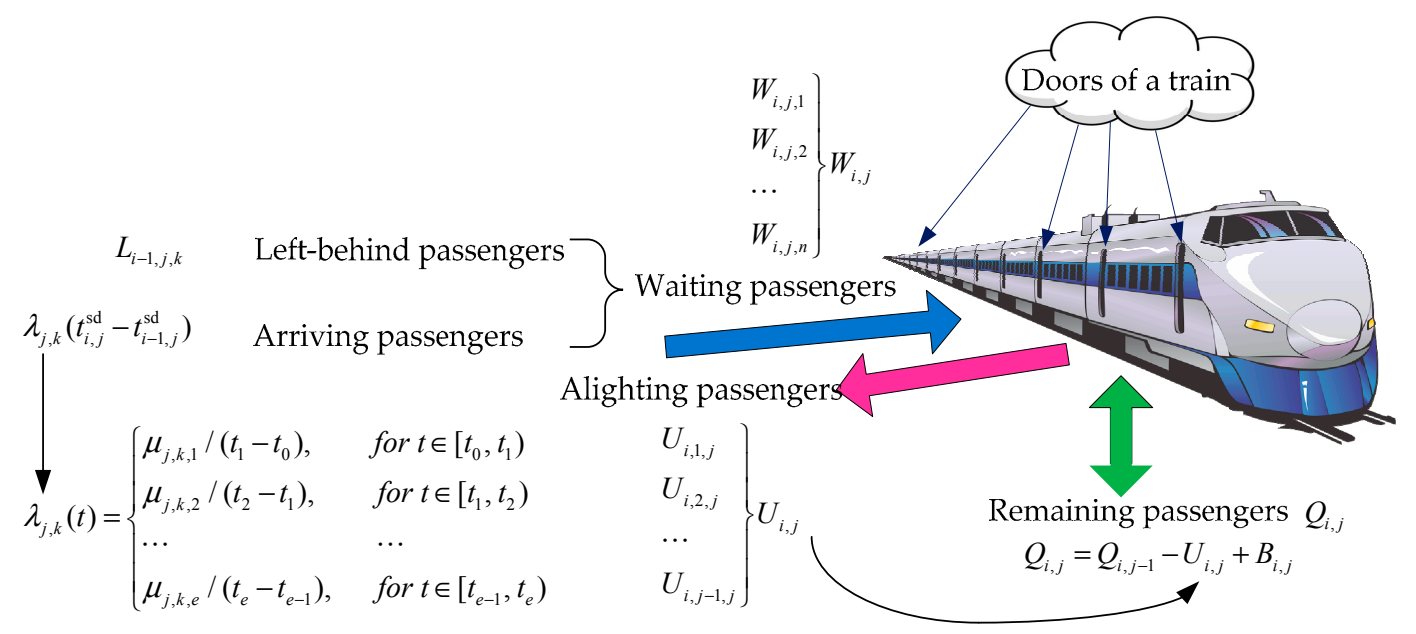

Figure 4. Illustration of passengers' activity information when using a metro train.

The number of waiting passengers $W_{i, j, k}$ at station $j$ desiring to alight at destination $k$ from train $i$ can be derived from:

$$
W_{i, j, k}=L_{i-1, j, k}+\lambda_{j, k}\left(t_{i, j}^{\mathrm{sd}}-t_{i-1, j}^{\mathrm{sd}}\right) i \geq 2, \forall j, k
$$

where $L_{i-1, j, k}$ is the number of waiting passengers left behind by train $i-1$ at station $j$ with destination $k$. $\lambda_{j, k}$ denotes the passenger flow arrival rate from station $j$ to station $k$. As introduced in Reference [29], the passenger flow arrival rates in sections is used to describe the passenger demand, which can be written as:

$$
\lambda_{j, k}(t)=\left\{\begin{array}{cc}
\sigma_{j, k, 1} /\left(t_{1}-t_{0}\right), & \text { for } t \in\left[t_{0}, t_{1}\right) \\
\sigma_{j, k, 2} /\left(t_{2}-t_{1}\right), & \text { for } t \in\left[t_{1}, t_{2}\right) \\
\cdots & \cdots \\
\sigma_{j, k, q} /\left(t_{q}-t_{q-1}\right), & \text { for } t \in\left[t_{q-1}, t_{q}\right) \\
\cdots & \cdots \\
\sigma_{j, k, e} /\left(t_{e}-t_{e-1}\right), & \text { for } t \in\left[t_{e-1}, t_{e}\right)
\end{array}\right.
$$

where the operation period $\left[t_{0}, t_{e}\right]$ is split into $e$ time slots with the splitting time instants $t_{0}, t_{1}, \ldots$, and $t_{e}, \sigma_{j, k, q}$ is the number of passengers travelling between station $j$ and $k$ in time slot $\left[t_{q-1}, t_{q}\right)$.

To account for the number of passengers alighting from train $i$ at station $j, U_{i, j}$ is given below:

$$
U_{i, j}=\sum_{f=1}^{j-1} U_{i, f, j} \forall f<j, f \in J
$$

where $U_{i, f, j}$ is the number of passengers boarding train $i$ at station $f$ and alighting at station $j$, which can be calculated as:

$$
U_{i, f, j}=W_{i, f, j}-L_{i, f, j}
$$

Moreover, the number of passengers travelling at train $i$ when the train departures from station $j$ is $Q_{i, j}$. The waiting passengers at a station will get on the train $i$ until the number of onboard passengers $B_{i, j}$ reaches its capacity $C$. After a train $i$ departs from a station, the number of remaining passengers $Q_{i, j}$ in the train is as follows in Equations (16)-(18):

$$
\begin{gathered}
Q_{i, j}=Q_{i, j-1}-U_{i, j}+B_{i, j} \\
B_{i, j}=\min \left\{Q_{i, j}^{\mathrm{rem}}, W_{i, j}\right\} \\
Q_{i, j}^{\text {rem }}=C-Q_{i, j-1}+U_{i, j}
\end{gathered}
$$


where $B_{i, j}$ is the number of passengers capable of boarding train $i$ at station $j$, which is the minimum remaining capacity $Q_{i, j}^{\text {rem }}$ of train $i$ at station $j$ and variable $W_{i, j}$.

During the rush hours, the number of left-behind passengers is formulated as follows:

$$
L_{i, j}=W_{i, j}-\min \left\{Q_{i, j}^{\mathrm{rem}}, W_{i, j}\right\}
$$

In order to fulfill assumption (5), actual dwell time $\tau_{i, j}^{\mathrm{a}}$ of train $i$ at station $j$ is defined as the maximum time between the minimized scheduled dwell time $\tau_{i, j, \min }^{\mathrm{s}}$ and the required time for the boarding and alighting passengers. In this paper, we use Equation (20) to estimate the actual dwell time $\tau_{i, j}^{\mathrm{a}}$. For Equation (20), a nonlinear function form has been proven availably [30]. The minimum dwell time is influenced by the number of passengers boarding $U_{i, j}$ and alighting from a $\operatorname{train} B_{i, j}$ :

$$
\tau_{i, j}^{\mathrm{a}}=\max \left\{\tau_{i, j, \min ^{\prime}}^{\mathrm{s}} \beta_{1}+\beta_{2} U_{i, j}+\beta_{3} B_{i, j}+\beta_{4}\left(\frac{W_{i, j}}{d}\right)^{3} B_{i, j}\right\}
$$

where $\beta_{1}, \beta_{2}, \beta_{3}$, and $\beta_{4}$ are fitting parameters and $d$ is the number of doors of a train.

As briefly stated in this section, the mathematical formulation is to find out the problem of the activity reality diverging from the real-time or historical information. In this problem, passenger activity information, especially in the oversaturated situations, is considered to be the important input. In the next section the robust approach is presented.

\section{Applying the Robust Approach to the Model}

The schedule-based timetable during the planning stage, generated by the above formulated mathematical model, is not sufficiently robust against unscheduled events. In the empirical case, the passenger congestion, accompanied by uncertainty, has a stochastic impact on the normal interaction. It is inevitable that the regular behaviors of boarding and alighting are disturbed creating a measurable delay. This is the key area in which a more reliable timetable would be beneficial under this kind of quantitative uncertainty by adjusting the buffer time.

The uncertainty, or deviation, of a scheduled timetable is associated with the estimated relationship between the delay and buffer time. If the minimum technological running time of a train is $3 \mathrm{~min}$, and the schedule running time of the same certain segment is $4 \mathrm{~min}$, we can infer that the buffer time is specified as $1 \mathrm{~min}$ by subtracting the two values. Based on this premise, the train would be behind schedule if it stopped at the previous station for more than $1 \mathrm{~min}$.

\subsection{Robust Mechanism}

The above-mentioned scheduled running time $t_{i, j-1, j}^{\mathrm{s}}$ is the sum of the expected value $E\left(t_{i, j-1, j}\right)$ of actual running time $t_{i, j-1, j}$ and the buffer time $x_{i, j-1, j}$ of train $i$ at the segment $e(j-1, j)$. We can obtain:

$$
\begin{gathered}
t_{i, j-1, j}^{\mathrm{s}}=E\left(t_{i, j-1, j}\right)+x_{i, j-1, j} \\
x_{i, j-1, j} \in\left[-\left(t_{i, j-1, j}^{\mathrm{s}, \max }-t_{i, j-1, j}^{\mathrm{s}, \min }\right),\left(t_{i, j-1, j}^{\mathrm{s}, \max }-t_{i, j-1, j}^{\mathrm{s}, \min }\right)\right]
\end{gathered}
$$

where $t_{i, j-1, j}$ is actual running time, $t_{i, j-1, j}^{\mathrm{s}, \max }$ and $t_{i, j-1, j}^{\mathrm{s}, \min }$ are the allowable maximum and minimum running times related to the technology level.

The deviation is derived from the difference between the scheduled departure time and the actual departure time.

$$
t_{i, j}^{\mathrm{d}}=t_{i, j}^{\mathrm{sd}}-t_{i, j}^{\mathrm{ad}}
$$

Clearly, train $i$ will depart from station $j$ later than the schedule if $t_{i, j}^{\mathrm{d}}<0$. Otherwise, $t_{i, j}^{\mathrm{d}}>0$ and it indicates the scheduled dwell time is too long and should be adjusted. 
During real operation, the drivers are expected to adjust the speed of the train to catch up to the planned timetable or reduce the delay if the actual departure time is disturbed. We can define the recovery period $t_{i, j-1, j}^{\mathrm{a}}$ as the expression in Equation (24).

$$
t_{i, j-1, j}^{\mathrm{a}}=\delta_{i, j-1, j}\left(t_{i, j-1}^{\mathrm{sd}}-t_{i, j-1}^{\mathrm{ad}}\right)
$$

where $\delta_{i, j-1, j}$ denotes the recovery factor for train $i$ at the segment $e(j-1, j)$ to achieve punctuality and $0 \leq \delta_{i, j-1, j} \leq 1$. For example, $\delta_{i, 1,2}=0$ indicates that the departure time of train $i$ is on time.

The adjusted running time is formulated in Equation (25) after the driver changes the speed:

$$
t_{i, j-1, j}^{\prime}=t_{i, j-1, j}+t_{i, j-1, j}^{\mathrm{a}}
$$

Similar to Equations (2) and (3), we can obtain the actual departure time of train $i$ at station $j$ :

$$
t_{i, j}^{\mathrm{ad}}=t_{i, j-1}^{\mathrm{ad}}+t_{i, j-1, j}^{\prime}+\tau_{i, j}^{\mathrm{a}}
$$

Thus, a major novelty of our model is the ability to deduce the recursion formula of the disturbed time $t_{i, j}^{\mathrm{d}}$, which is accounted as follows:

$$
\begin{aligned}
t_{i, j}^{\mathrm{d}} & =t_{i, j}^{\mathrm{sd}}-t_{i, j}^{\mathrm{ad}}=t_{i, j-1}^{\mathrm{sd}}+t_{i, j-1, j}^{\mathrm{s}}+\tau_{i, j}^{\mathrm{s}}-\left(t_{i, j-1}^{\mathrm{ad}}+t_{i, j-1, j}+t_{i, j-1, j}^{\mathrm{a}}+\tau_{i, j}^{\mathrm{a}}\right) \\
& =\left(t_{i, j-1}^{\mathrm{s}}-t_{i, j-1}^{\mathrm{ad}}\right)+\left(t_{i, j}^{\mathrm{s}}-1, j\right. \\
& =\left(1-\delta_{i, j, j-1, j}\right)\left(t_{i, j-1}^{\mathrm{sd}}-t_{i, j-1}^{\mathrm{ad}}\right)+\left(t_{i, j-1, j}^{\mathrm{s}}-t_{i, j-1, j}\left(t_{i, j-1}^{\mathrm{sd}}-t_{i, j-1}^{\mathrm{ad}}\right)+\tau_{i, j}^{\mathrm{s}}-\tau_{i, j}^{\mathrm{a}}-\tau_{i, j}^{\mathrm{a}}\right. \\
& =\left(1-\delta_{i, j-1, j}\right) t_{i, j-1}^{\mathrm{d}}+\left(t_{i, j-1, j}^{\mathrm{s}}-t_{i, j-1, j}\right)+\tau_{i, j}^{\mathrm{s}}-\tau_{i, j}^{\mathrm{a}}
\end{aligned}
$$

Adding Equation (21), the above formulation could be rewritten as:

$$
t_{i, j}^{\mathrm{d}}=\left(1-\delta_{i, j-1, j}\right) t_{i, j-1}^{\mathrm{d}}+\left[E\left(t_{i, j-1, j}\right)+x_{i, j-1, j}-t_{i, j-1, j}\right]+\tau_{i, j}^{\mathrm{s}}-\tau_{i, j}^{\mathrm{a}}
$$

The departure time from the original station is assumed to be on time, so:

$$
t_{i, 1}^{\mathrm{d}}=0
$$

The decision variable candidates in the model are:

- Actual running time set of train $i$ at the segment $e(j-1, j): t=\left\{t_{i, j-1, j} \mid i \in I, j \in J\right\}$

- Recovery factor set for train $i$ at the segment $e(j-1, j): \delta=\left\{\delta_{i, j-1, j} \mid i \in I, j \in J\right\}$

- Actual dwell time set of train $i$ at station $j: \tau=\left\{\tau_{i, j}^{a} \mid i \in I, j \in J\right\}$

- Buffer time set of train $i$ at the segment $e(j-1, j): x=\left\{x_{i, j-1, j} \mid i \in I, j \in J\right\}$

In this paper, we consider buffer time set as decision variables on a metro line. In the period of time under consideration, the headway and departure time from the original station are fixed. Besides, another three sets (actual running time set, recovery factor set and actual dwell time set) are used as parameters derived from real-time information or as weighted parameters.

\subsection{Objective}

Disturbed time $t_{i, j}^{\mathrm{d}}$ is the function of the decision variables $t_{i, j}^{\mathrm{d}}(t, \delta, \tau, x)$. Sets $t$ and $\tau$ can be calibrated or estimated from historical data gathered from the AFC system. Set $\delta$ was acquired from repeated experiments. Thus, sets $t, \tau$, and $\delta$ are the inputs for the model and set $x$ is the output results.

Let parameters $\gamma_{1}$ and $\gamma_{2}$ be penalties on delay $\left(t_{i, j}^{\mathrm{d}}<0\right)$ and ahead $\left(t_{i, j}^{\mathrm{d}}>0\right)$ of scheduled departure time, respectively. The values of both parameters are determined by train dispatchers according to their preferences. Their impact on the solution will be evaluated in the section with the numerical example. The penalty ratio $\left(\gamma_{1} / \gamma_{2}\right)$ determines its performance. To evaluate the impact 
of parameters $\gamma_{1}$ and $\gamma_{2}$, Yan et al. further presented the following two special scenarios of the robust optimization model by setting $\gamma_{1}=2 \gamma_{2}$ and $\gamma_{1}=0.5 \gamma_{2}$ [31]. With different preferences, some dispatchers regard this as undesirable service expectations derived from earlier arrival, while the alternative view is that later arrival causes more inconvenience to passengers and extra operating cost to them [32,33]. In the situation when $\gamma_{1}=\gamma_{2}$, earlier arrival and later arrival are assumed to be equivalently penalized. When $\gamma_{1}=2 \gamma_{2}$, earlier arrival is assumed to be more undesirable than later arrival. The penalty of a unit of schedule deviation for earlier arrival is assumed to be twice as much as that of a unit of schedule deviation for later arrival. In a similar way, an opposite evaluation can also be taken into account from $\gamma_{1}=0.5 \gamma_{2}$, with the later arrival more penalized. Thus, we can follow a generalized timetabling deviation function in Equation (30):

$$
D_{i, j}\left(t, \delta, \tau, x, \gamma_{1}, \gamma_{2}\right)=\gamma_{1} \max \left\{t_{i, j}^{\mathrm{d}}(t, \delta, \tau, x), 0\right\}+\gamma_{2} \max \left\{-t_{i, j}^{\mathrm{d}}(t, \delta, \tau, x), 0\right\}
$$

For a robust timetable combined with the passengers' activity information, reliability is usually enhanced with increasing the match between the scheduled plan and the actual operation. The punctuality is performed on the adjustment on the set of buffer time. If the buffer time is not long enough, the drivers are not able to catch up to the original schedule. Otherwise, the frequency of service is not satisfactory to passengers suffering from the longer waiting times. The later or earlier conditions contribute side effects on the reliable timetable. Therefore, the objective functions are given as follows:

$$
\Omega=\min \sum_{i=1}^{m} \sum_{j=2}^{n}\left|D_{i, j}\left(t, \delta, \tau, x, \gamma_{1}, \gamma_{2}\right)\right|
$$

The details of RTM are addressed for a metro line with passenger activity information, which take into account congestion and buffer time adjustments. The main objective and essential constraints are described mathematically. In practice, when applying the proposed model to empirical cases, the available solution algorithm is necessary and presented in next section.

\section{Solution Algorithm}

By precisely accounting for the passenger demand records, available resources, and practical regulations, the above section describes a comprehensive model to capture the timetabling constraints, passengers' activity information, and enhancement of the robust approach on a metro line. The developed model, a mixed integer nonlinear programming (MINLP) model with complicated constraints, cannot be solved by any open-source exact solvers. The objective variable contains two dimensions: $i$ and $j$. First, for the operating period of train $i$, we should estimate and handle the passengers' activity information. Next, at the second step, we need to figure out penalties on delay $\left(t_{i, j}^{\mathrm{d}}<0\right)$ and ahead $\left(t_{i, j}^{\mathrm{d}}>0\right)$ of scheduled departure time, respectively. Obviously, it is repeated to seek the optimal solution rather than one-time input/output. However, the input set has to be judged on whether delay or ahead of schedule at each segment $\left(\left\{x_{i, j-1, j}, i \in I, j \in J\right\}\right)$. These calculated features (i.e., mixed and nonlinear features) of our model requires the algorithm to be experimental seeking tool and repeated optimization solver. Based on the above characteristics of computation, it is available to apply a heuristic method such as GA. To further provide insights into the train timetabling problem, considering loading capacity, we demonstrate the process of adjusting the buffer times for developing a robust timetabling plan.

Step 0 (Initialization): Calculate the matrix of actual dwell time based on the demand distribution (origin-destination, OD) and Equations (11)-(20).

Step 1 (Input): Input the set of actual running $t$ and recovery factor $\delta$. Parameters $\gamma_{1}$ and $\gamma_{2}$ are estimated and one arbitrary value $\Omega^{0}$ is assumed as the upper bound of the objective. Let $g=1$ and we set current objective value $\Omega^{g}=\Omega^{0}$. 
Step 2 (Encode gens): Encode buffer time $x_{i, j-1, j}$ of segment $e(j-1, j)$ for train $i$ as a generation belonging to the solution set (i.e., a chromosome in GA). At the same time, it is also the decision variable. The set of genes $\left\{x_{i, j-1, j}, i \in I, j \in J\right\}$ is defined as chromosome.

Step 3 (Crossover and mutation operators): Since the decision variables of the proposed model are $x_{i, j-1, j}$, they are used as genes for any chromosome in the GA. Decision variable $x_{i, j-1, j}$ is generated at random by capturing from domain of definition $\left[-\left(t_{i, j-1, j}^{\mathrm{s}, \max }-t_{i, j-1, j}^{\mathrm{s}, \min }\right),\left(t_{i, j-1, j}^{\mathrm{s}, \max }-\right.\right.$ $\left.\left.t_{i, j-1, j}^{\mathrm{s}, \mathrm{min}}\right)\right]$, and the first chromosome was created randomly. For example, for one metro line of 13 located stations, some sample chromosomes are shown in Figure 5.

The most common operators used in a genetic algorithm are:

- Crossover

- Mutation

$$
[0,0,0,0,0,0,0,0,0,0,0,0,0], \quad[4,9,2,16,1,23,8,1,5,6,7,10,-5]
$$

Figure 5. Sample chromosomes for one example.

Both operators are used in the algorithm in this research. In linear crossover, a gene value is replaced by the value of the same gene in another chromosome (see Figure 6). It is clear that linear crossover will result in no conflicts according to the bounds of the variables $x_{i, j-1, j}$, which is the reason behind this decision.

$$
\begin{aligned}
& \left\{\begin{array}{l}
{[0,0,0,0,0,0,0,0,0,0,0,0,0],} \\
{[4,9,2,16,1,23,8,1,5,6,7,10,-5]}
\end{array}\right. \\
& \text { Crossover } \\
& \left\{\begin{array}{l}
{[0,0,0,0,1,0,0,0,5,0,0,10,0], \text { Mutation }} \\
{[4,9,2,16,0,23,8,1,0,6,7,0,-4]}
\end{array}\right.
\end{aligned}
$$

Figure 6. Examples of crossover operation for the sample chromosomes of the above example.

The crossover operation usually results in local optimum solutions. Thus, a mutation operation is used to escape local optimums. In many genetic algorithms, the variables are binary, and mutations indicate the change of a variable from 0 to 1 or vice versa. However, here, we have general integer variables. For a gene $x_{i, j-1, j}$ with any possible value, a random number is generated in the range of $\left[-\left(t_{i, j-1, j}^{\mathrm{s}, \max }-t_{i, j-1, j}^{\mathrm{s}, \min }\right),\left(t_{i, j-1, j}^{\mathrm{s}, \max }-t_{i, j-1, j}^{\mathrm{s}, \min }\right)\right]$. By replacing this number with $x_{i, j-1, j}$, a new value for the variable is created. Next, the objective variables $\Omega$ are calculated with crossover and mutation operators (described in detail in Reference $[13,25]$ ) based on Equations (21)-(30). If $\Omega<\Omega^{g}$, then $\Omega=\Omega^{g}$; otherwise, continue to search.

Step 4 (Fitness evaluation): Fitness evaluation is used to measure the goodness of individual candidates. The fitness value in the GA is calculated by Equation (32). The genetic search prefers individuals with higher fitness:

$$
\Omega=\min \sum_{i=1}^{m} \sum_{j=2}^{n}\left|D_{i, j}\left(x_{i, j-1, j}\right)\right|
$$


Step 5 (Stopping criterion): We can obtain the optimization solution $\left\{x_{i, j-1, j}, i \in I, j \in J\right\}$ within the convergence, if no improvement can be made in the specified time and stated number of generations.

The stopping criteria for termination of the genetic algorithm are defined as follows:

- The best fitness function does not change after a given number of iterations.

- The difference between the best and worst solutions in a chromosome is less than a given value, i.e., $1 \%$.

- The algorithm reaches a maximum number of iterations.

We present a solution algorithm for the RTM described in the previous section. The proposed MINLP mathematical model consists of a large number of variables. Therefore, in this section a heuristic algorithm (GA) is proposed to yield satisfactory results.

\section{Numerical Example}

Considering the passengers' activity information, the proposed solution procedure is applied to design the robust timetable for the Batong Line of Beijing, China, which consists of 13 stations (Figure 7). The technological operating parameters of a metro train are given in Table 1. The specific input data on the segments are summarized in Table 2, and the passenger flow arrival rate in the downstream direction of the Batong Line is provided in Table 3. The value of crossover rate and mutation rate are 0.2 and 0.03, respectively. In this real example, one hour (18:30:00-19:30:00) is selected from the operational period with the varying weighting values $\gamma_{1}$ and $\gamma_{2}$ for three scenarios. The best-adjusted solution is achieved by our proposed model based on GA, described by MATLAB. All the experiments are performed on a computer with Intel(R) Core(TM) i5 CPU running at $2.90 \mathrm{GHz}$ and 4.00 GB of RAM, using Microsoft Windows 7 (64 bit) OS. To reach the optimal solution of $54 \mathrm{~min}$ for the objective function, 831 iterations were performed by GA based approximate 20 trials with convergent termination.

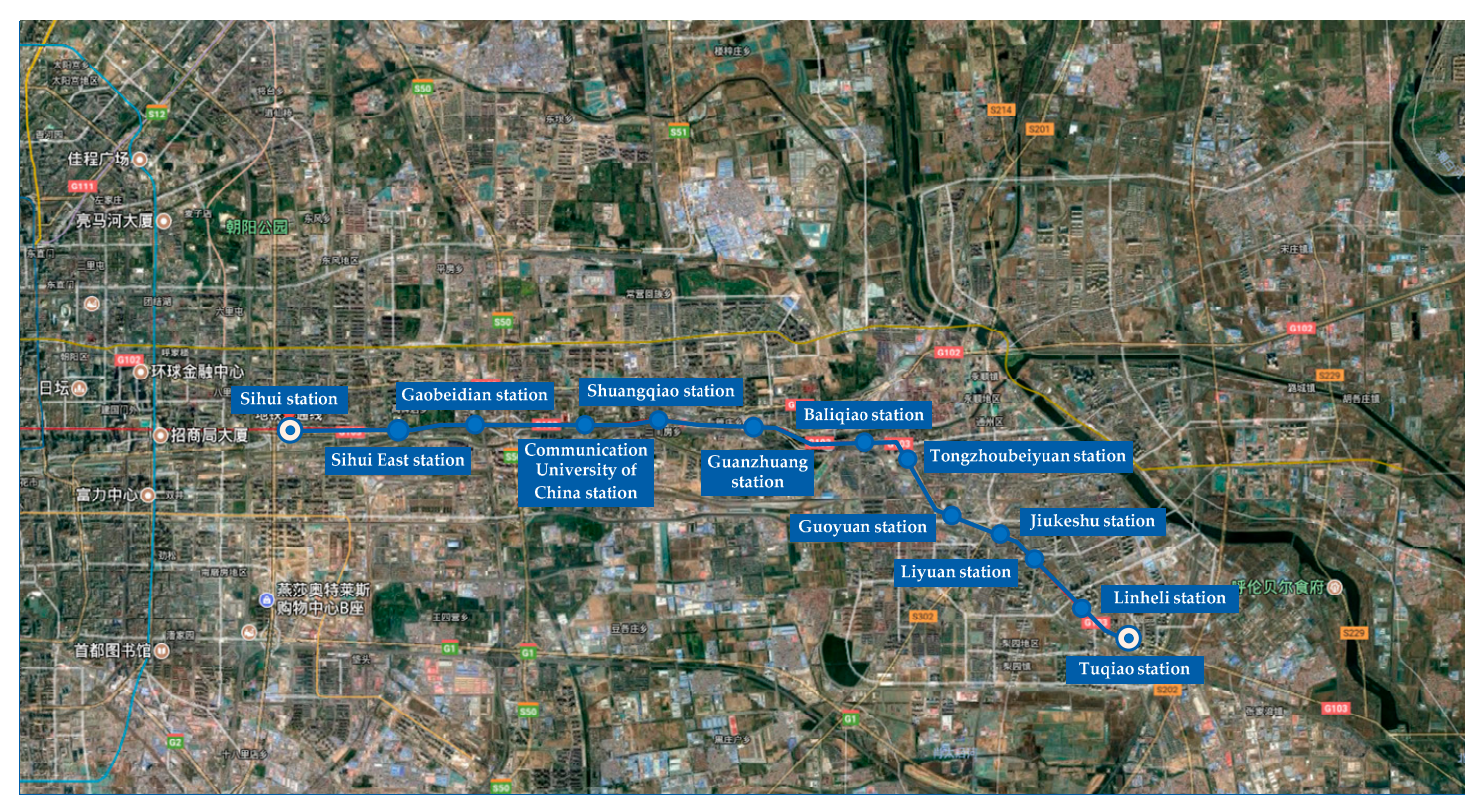

Figure 7. Robust mechanism of timetabling process according to the distributed time. 
Table 1. Model parameters following appearing orders.

\begin{tabular}{ccc}
\hline Parameters Input & Symbols & Value and Unit \\
\hline Loading capacity of a train & $C$ & $1468 \mathrm{pax} \cdot \mathrm{train}^{-1}$ \\
Maximum speeds of a train & $v_{i, j-1, j}^{\max }$ & $13.9 \mathrm{~m} \cdot \mathrm{s}^{-1}$ \\
Minimum speeds of a train & $v_{i, j-1, j}^{\mathrm{min}}$ & $5.6 \mathrm{~m} \cdot \mathrm{s}^{-1}$ \\
Acceleration of a train & $a_{i, j-1, j}^{\text {acc }}$ & $0.8 \mathrm{~m} \cdot \mathrm{s}^{-2}$ \\
Deceleration of a train & $a_{i, j-1, j}^{\text {dec }}$ & $0.7 \mathrm{~m} \cdot \mathrm{s}^{-2}$ \\
Minimum dwell time & $\tau_{i, j, \min }^{\mathrm{s}}$ & $30 \mathrm{~s}$ \\
Number of doors of a train & $d$ & 24 doors $\cdot \operatorname{train}^{-1}$ \\
\hline & $\beta_{1}$ & 4.002 \\
Fitting parameters & $\beta_{2}$ & 0.047 \\
& $\beta_{3}$ & 0.051 \\
& $\beta_{4}$ & 0.010 \\
\hline
\end{tabular}

Table 2. The specific input data on segments.

\begin{tabular}{|c|c|c|c|c|}
\hline Segments & $\begin{array}{l}\text { Length of Segments } \\
\qquad l(j-1, j) / \mathrm{m}\end{array}$ & $\begin{array}{c}\text { Expected Values of } \\
\text { Actual Running Time } \\
\text { of a Train } E\left(t_{i, j-1, j}\right) / \mathrm{s}\end{array}$ & $\begin{array}{c}\text { Minimum } \\
\text { Running Time of } \\
\text { a Train } t_{i, j-1, j}^{s, m i n} / \mathbf{s}\end{array}$ & $\begin{array}{c}\text { Maximum } \\
\text { Running Time of } \\
\text { a Train } s_{i, j-1, j}^{\max } / \mathrm{s}\end{array}$ \\
\hline $1-2$ & 776 & 174 & 158 & 214 \\
\hline $2-3$ & 1257 & 223 & 190 & 262 \\
\hline $3-4$ & 1225 & 214 & 183 & 245 \\
\hline $4-5$ & 990 & 202 & 171 & 249 \\
\hline $5-6$ & 1465 & 258 & 223 & 297 \\
\hline $6-7$ & 1700 & 241 & 208 & 283 \\
\hline $7-8$ & 1763 & 250 & 213 & 286 \\
\hline $8-9$ & 1912 & 239 & 201 & 277 \\
\hline $9-10$ & 1894 & 239 & 206 & 282 \\
\hline 10-11 & 2002 & 232 & 201 & 279 \\
\hline $11-12$ & 1375 & 229 & 198 & 265 \\
\hline $12-13$ & 1715 & 227 & 194 & 261 \\
\hline
\end{tabular}

Note: 1-Tuqiao station; 2-Linheli station; 3-Liyuan station; 4-Jiukeshu station; 5-Guoyuan station; 6-Tongzhoubeiyuan station; 7-Baliqiao station; 8-Guanzhuang station; 9-Shuangqiao station; 10-Communication University of China station; 11-Gaobeidian station; 12-Sihui East station; 13-Sihui station.

Table 3. Passenger flow arrival rate at downstream direction of Batong Line. Unit: $\mathrm{pax} \cdot \mathrm{s}^{-1}$.

\begin{tabular}{cccccccccccccc}
\hline Station & $\mathbf{1}$ & $\mathbf{2}$ & $\mathbf{3}$ & $\mathbf{4}$ & $\mathbf{5}$ & $\mathbf{6}$ & $\mathbf{7}$ & $\mathbf{8}$ & $\mathbf{9}$ & $\mathbf{1 0}$ & $\mathbf{1 1}$ & $\mathbf{1 2}$ & $\mathbf{1 3}$ \\
\hline 1 & 0 & 0.15 & 0.55 & 0.35 & 0.15 & 0.55 & 0.35 & 0.72 & 0.45 & 0.14 & 0.35 & 0.25 & 0.35 \\
2 & 0 & 0 & 0.35 & 0.45 & 0.38 & 0.25 & 0.25 & 0.45 & 0.28 & 0.42 & 0.08 & 0.20 & 0.25 \\
3 & 0 & 0 & 0 & 0.36 & 0.15 & 0.15 & 0.10 & 0.26 & 0.25 & 0.16 & 0.40 & 0.15 & 0.36 \\
4 & 0 & 0 & 0 & 0 & 0.20 & 0.12 & 0.18 & 0.15 & 0.10 & 0.37 & 0.35 & 0.10 & 0.48 \\
5 & 0 & 0 & 0 & 0 & 0 & 0.18 & 0.23 & 0.18 & 0.26 & 0.28 & 0.28 & 0.20 & 0.38 \\
6 & 0 & 0 & 0 & 0 & 0 & 0 & 0.21 & 0.26 & 0.38 & 0.35 & 0.16 & 0.15 & 0.24 \\
7 & 0 & 0 & 0 & 0 & 0 & 0 & 0 & 0.03 & 0.20 & 0.20 & 0.10 & 0.24 & 0.35 \\
8 & 0 & 0 & 0 & 0 & 0 & 0 & 0 & 0 & 0.08 & 0.30 & 0.20 & 0.23 & 0.24 \\
9 & 0 & 0 & 0 & 0 & 0 & 0 & 0 & 0 & 0 & 0.15 & 0.36 & 0.35 & 0.31 \\
10 & 0 & 0 & 0 & 0 & 0 & 0 & 0 & 0 & 0 & 0 & 0.42 & 0.41 & 0.28 \\
11 & 0 & 0 & 0 & 0 & 0 & 0 & 0 & 0 & 0 & 0 & 0 & 0.22 & 0.27 \\
12 & 0 & 0 & 0 & 0 & 0 & 0 & 0 & 0 & 0 & 0 & 0 & 0 & 0.31 \\
13 & 0 & 0 & 0 & 0 & 0 & 0 & 0 & 0 & 0 & 0 & 0 & 0 & 0 \\
\hline
\end{tabular}

Based on the actual dwell time as shown in Table 4, we can obtain the optimized results in Table 5. The recovery time and scheduled running time in Table 6 are essentially consistent with the passengers' activity information. In conducting a fair comparison between three scenarios in Table 7, the performance of the robust assessment of timetabling turns out to be available at different penalties. The analysis of the mechanism is given as follows: 
(i) Scenario 1: If the penalties for both delays and being ahead of schedule are identical, the recovery time (the sum of absolute value of buffer time) is shortest at $234 \mathrm{~s}$. At the same time, the scheduled running time is the shortest at $2804 \mathrm{~s}$.

(ii) Scenario 2: The recovery time is longest at $283 \mathrm{~s}$ when the penalty for a delay is perceived to be greater than that for being ahead of schedule. The scheduled running time is the longest at $3300 \mathrm{~s}$.

(iii) Scenario 3: It is assumed that a delay might be less frustrating than that of being ahead of schedule. As a result, we find a recovery time of $254 \mathrm{~s}$ and a scheduled running time of $3279 \mathrm{~s}$.

Table 4. Actual dwell time of a train at each station. Unit: s.

\begin{tabular}{cccccccccccccc}
\hline Station & $\mathbf{1}$ & $\mathbf{2}$ & $\mathbf{3}$ & $\mathbf{4}$ & $\mathbf{5}$ & $\mathbf{6}$ & $\mathbf{7}$ & $\mathbf{8}$ & $\mathbf{9}$ & $\mathbf{1 0}$ & $\mathbf{1 1}$ & $\mathbf{1 2}$ & $\mathbf{1 3}$ \\
\hline Actual dwell time & 30.0 & 30.0 & 38.4 & 37.4 & 36.4 & 29.5 & 34.2 & 45.1 & 30.8 & 31.4 & 30.7 & 31.8 & 34.2 \\
\hline
\end{tabular}

Table 5. Optimized results. Unit: s.

\begin{tabular}{ccccccc}
\hline \multirow{2}{*}{ Segment } & \multicolumn{2}{c}{ Scenario $\mathbf{1}\left(\gamma_{\mathbf{1}}=\mathbf{1}, \gamma_{\mathbf{2}}=\mathbf{1}\right)$} & \multicolumn{2}{c}{ Scenario $\mathbf{2}\left(\gamma_{\mathbf{1}}=\mathbf{2}, \gamma_{\mathbf{2}}=\mathbf{1}\right)$} & \multicolumn{2}{c}{ Scenario $\mathbf{3}\left(\gamma_{\mathbf{1}}=\mathbf{0 . 5}, \gamma_{\mathbf{2}}=\mathbf{1}\right)$} \\
\cline { 2 - 7 } & Buffer Time & $\begin{array}{c}\text { Scheduled } \\
\text { Running Time }\end{array}$ & Buffer Time & $\begin{array}{c}\text { Scheduled } \\
\text { Running Time }\end{array}$ & Buffer Time & $\begin{array}{c}\text { Scheduled } \\
\text { Running Time }\end{array}$ \\
\hline $1-2$ & -17 & 157 & 23 & 197 & -11 & 163 \\
$2-3$ & -8 & 215 & 35 & 258 & 15 & 238 \\
$3-4$ & 5 & 219 & -18 & 196 & 32 & 246 \\
$4-5$ & 12 & 214 & 34 & 236 & 44 & 246 \\
$5-6$ & 20 & 278 & -12 & 246 & 12 & 270 \\
$6-7$ & 42 & 283 & 25 & 266 & -18 & 223 \\
$7-8$ & -14 & 236 & -30 & 220 & 11 & 261 \\
$8-9$ & 22 & 261 & 21 & 260 & 25 & 264 \\
$9-10$ & 25 & 264 & 38 & 277 & 13 & 252 \\
$10-11$ & -5 & 227 & 25 & 257 & 28 & 260 \\
$11-12$ & -35 & 194 & 9 & 238 & -27 & 222 \\
$12-13$ & 29 & 256 & 13 & 240 & 18 & 245 \\
\hline
\end{tabular}

Table 6. Recovery time and scheduled running time. Unit: s.

\begin{tabular}{ccc}
\hline Scenario & Recovery Time & Scheduled Running Time \\
\hline Scenario 1 & 234 & 2804 \\
Scenario 2 & 283 & 3300 \\
Scenario 3 & 254 & 3279 \\
\hline
\end{tabular}

Table 7. The generated robust timetable based on passengers activity information. Unit: s.

\begin{tabular}{cccc}
\hline \multirow{2}{*}{ Station } & \multicolumn{3}{c}{ Departure Time } \\
\cline { 2 - 4 } & Scenario $\mathbf{1}\left(\boldsymbol{\gamma}_{\mathbf{1}}=\mathbf{1}, \boldsymbol{\gamma}_{\mathbf{2}}=\mathbf{1}\right)$ & Scenario $\mathbf{2}\left(\boldsymbol{\gamma}_{\mathbf{1}}=\mathbf{2}, \boldsymbol{\gamma}_{\mathbf{2}}=\mathbf{1}\right)$ & Scenario $\mathbf{3}\left(\boldsymbol{\gamma}_{\mathbf{1}}=\mathbf{0 . 5}, \boldsymbol{\gamma}_{\mathbf{2}}=\mathbf{1}\right)$ \\
\hline 1 & $18: 30: 00$ & $18: 30: 00$ & $18: 30: 00$ \\
2 & $18: 33: 07$ & $18: 33: 47$ & $18: 33: 13$ \\
3 & $18: 37: 21$ & $18: 38: 44$ & $18: 37: 50$ \\
4 & $18: 41: 37$ & $18: 42: 47$ & $18: 42: 33$ \\
5 & $18: 45: 47$ & $18: 47: 09$ & $18: 47: 15$ \\
6 & $18: 49: 56$ & $18: 51: 45$ & $18: 52: 15$ \\
7 & $18: 55: 13$ & $18: 56: 45$ & $18: 56: 32$ \\
8 & $18: 59: 54$ & $19: 01: 10$ & $19: 01: 38$ \\
9 & $19: 04: 46$ & $19: 06: 01$ & $19: 06: 33$ \\
10 & $19: 09: 42$ & $19: 11: 10$ & $19: 11: 17$ \\
11 & $19: 14: 00$ & $19: 15: 57$ & $19: 16: 07$ \\
12 & $19: 17: 46$ & $19: 20: 27$ & $19: 20: 01$ \\
13 & $19: 22: 36$ & $19: 25: 01$ & $19: 24: 40$ \\
\hline
\end{tabular}


From the above discussions, we concluded that if the dispatchers are supposed to generate a robust timetabling plan by using the proposed model, they should adjust the parameters $\gamma_{1}$ and $\gamma_{2}$ as a response for the preferred scenarios. In the empirical cases, being ahead of schedule usually has the disadvantage of a much lower loading factor than normal operation because most passengers are on time. On the other hand, the long time for waiting tends to be disappointing and causes complaints, which leads to impatient passengers choosing an alternative such as private cars.

\section{Extension}

To the authors' knowledge, "time control point strategy" was first put forward and applied to transportation systems in [31]. However, this strategy is only proven with significant feasibility for train operations. In this section, we combined a time control point strategy into robust timetabling and this benefited the punctuality of major stations, such as transfer stations and hub stations, on a metro line.

In the above-proposed model, the buffer times at all stations were adjusted in order to reach the required robustness in the metro timetabling problem. In our work, a shift in enhancing robust timetable was used to reduce the total delay or advancement compared with the scheduled plan. However, it is not necessary for the departures from all stations to be guaranteed completely on time. Perhaps the dispatchers are not capable of ensuring the timeliness of all stations due to their stochastic and uncertain nature. Here, we introduce the time control point strategy into our model to improve the punctuality at major stations.

A virtual level is introduced corresponding to the schedule level based on an identical timeline shown in Figure 8. The time control points are distributed on this timeline in terms of formulating the new spatial and temporal distribution. These points correspond to the selected major stations which are supposed to guarantee timeliness under certain conditions. The set of points $R \subset J$ refining the station set. Now the zone between two adjacent stations includes more than two segments. Generally, the running time of this zone is accounted by the sum of running times of these several segments. In other words, the time control points are still the real stations between which some stations as relatively unimportant ones are not able to achieve their punctuality.

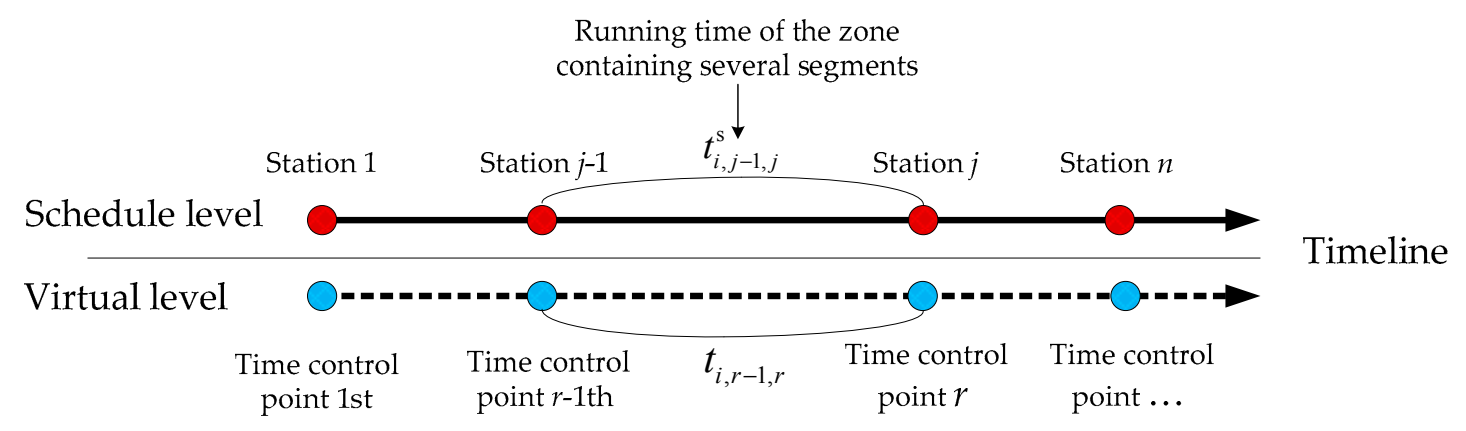

Figure 8. Illustration of time control point strategy.

This extensive research was conducted according to the above example, in which four time control points dealt with the virtual level corresponding to the 1st, 6st, 10st, and 13st stations on the scheduled level. By using the extension, we can reformulate the running time of the zone: $t_{i, 1,2}^{\text {virtual }}=$ $t_{i, 1,2}+t_{i, 2,3}+t_{i, 3,4}+t_{i, 4,5}+t_{i, 5,6}, t_{i, 2,3}^{\text {virtual }}=t_{i, 6,7}+t_{i, 7,8}+t_{i, 8,9}+t_{i, 9,10}, t_{i, 3,4}^{\text {virtual }}=t_{i, 10,11}+t_{i, 11,12}+t_{i, 12,13}$. The solution is acquired by our proposed approach by combining it with the time control point strategy in Table 8. For a schedule-based metro operation system, reliability of time control points is usually defined as the match between the scheduled plan (especially the scheduled departure time) and the actual operation. On-time performance of these determined points depends on how metro operation is able to match the transit schedule throughout our above-proposed modeling formulations. By this 
means of using our extension, it also proves that the usability of our model covers the whole or partial stations to reach different degrees of robustness. Besides, considering the special characteristics of RTM problem, established constraints can be used as needed. The examples of the extension may read: the subset of buffer times allocated to each zone between adjacent time control points can be handled by our model and solution algorithm; at present, one zone concludes at least two segments and three stations.

Table 8. Solution based on time control point strategy. Unit: s.

\begin{tabular}{ccccccc}
\hline Zone & \multicolumn{2}{c}{ Scenario $\mathbf{1}\left(\gamma_{\mathbf{1}}=\mathbf{1}, \gamma_{\mathbf{2}}=\mathbf{1}\right)$} & \multicolumn{2}{c}{ Scenario $\mathbf{2}\left(\gamma_{\mathbf{1}}=\mathbf{2}, \gamma_{\mathbf{2}}=\mathbf{1}\right)$} & \multicolumn{2}{c}{ Scenario $\mathbf{3}\left(\gamma_{\mathbf{1}}=\mathbf{0 . 5}, \gamma_{\mathbf{2}}=\mathbf{1}\right)$} \\
\cline { 2 - 7 } & Buffer Time & $\begin{array}{c}\text { Scheduled } \\
\text { Running Time }\end{array}$ & Buffer Time & $\begin{array}{c}\text { Scheduled } \\
\text { Running Time }\end{array}$ & Buffer Time & $\begin{array}{c}\text { Scheduled } \\
\text { Running Time }\end{array}$ \\
\hline $1-2$ & 4 & 1104 & -17 & 1083 & -32 & 1068 \\
$2-3$ & -23 & 977 & 41 & 1041 & 19 & 1019 \\
$3-4$ & 16 & 738 & 65 & 787 & 24 & 746 \\
\hline
\end{tabular}

From the results shown in Table 8, we compared it with the solution derived in Section 5 for all stations, in cases where the amount of buffer time exhibited a significant variation. In these experiments, the three scenarios showed a decrease of $18 \%, 57 \%$, and $30 \%$ respectively, compared to the previous solution. It can be noted that the amount of buffer time is reduced only if partial major stations are adjusted, rather than entire stations, along the corridor. However, the scheduled running time shows no obvious changes, which is due to the trade-off on adjusting buffer times between early and late departures, where the recovery time is relatively short without sharply fluctuating the end-to-end travel time.

Several experiments were conducted to test the model for different situations. The analysis focused on the key results: the optimal values of the buffer times and scheduled running time under normal operation and with the time control point strategy. This implies that the time control point strategy is a good option for dealing with the necessity of timeliness for specific stations.

\section{Conclusions}

In this paper, we introduced a RMT for a metro line with passengers' activity information, in which the load capacity constraint was considered. The robust timetable was rescheduled with the aim of enhancing its ability to withstand unscheduled events resulting from the congestion. Our proposed model is specially adapted to solving deviation problems during rush hours. In terms of historical demand data, the interactions between the boarding and alighting times, and scheduled dwell time, are explored so that a feasibly robust timetable is generated at the planning stage.

In our formulated model, the objective of minimizing the deviation from the scheduled timetable is a novel contribution. Passenger activity information for the real issues was analyzed to have a quantitative assessment of the delays or being ahead of the scheduled plan. The appearance of a lack of punctuality leads to adding the concept of buffer time. If the buffer time is so short, the planned schedule is not able to recover back to the original. Conversely, it might be too long to satisfy the LOS with an insufficient frequency of service. The late or early conditions contribute side effects on the reliable timetable. The reasonable adjustment of buffer time is paid significant attention in our modeling work.

GA is designed to solve the presented model because it is a MINLP model with the mathematical properties of large-scale search and combination explosion at enumerative calculation. Based on the real case of the Batong Line of the Beijing Metro, our approach is not only capable of improving the robustness for one specified station, but could also be applied to obtain better punctuality of running time between two major stations by using the time control point strategy. However, the essential long-term data collection is scarce, which will be completed in further research. As one problem of the 
comprehensive robust timetabling approach, a stop-skipping scheme will be added into the studied schedule towards an ongoing issue, which will be studied in future work.

Acknowledgments: This research is supported by the National Basic Research Program of China (No. 2012CB725403).

Author Contributions: Lai Wei and Zhenzhou Yuan provided relevant information, discussed the data, and corrected the manuscript; and Lai Wei revised the manuscript. All authors have read and approved the final manuscript. All authors would also like to thank Enjian Yao and Yihui Wang for their advice on this paper.

Conflicts of Interest: There is no conflict of interest related to the content of this paper.

\section{References}

1. Takeuchi, H.; Goodman, C.; Sone, S. Moving block signaling dynamics: Performance measures and re-starting queued electric trains. Proc. Inst. Elect. Eng.-Elect. Power Appl. 2003, 150, 483-492. [CrossRef]

2. Shafia, M.A.; Aghaee, M.P.; Sadjadi, S.J.; Jamili, A. Robust train timetabling problem: Mathematical model and branch and bound algorithm. IEEE Trans. Intell. Transp. Syst. 2012, 13, 307-317. [CrossRef]

3. Newell, G.F. Dispatching policies for a transportation route. Transp. Sci. 1971, 5, 91-105. [CrossRef]

4. Osuna, E.E.; Newell, G.F. Control strategies for an idealized public transportation system. Transp. Sci. 1972, 6, 52-72. [CrossRef]

5. De Palma, A.; Lindsey, R. Optimal timetables for public transportation. Transp. Res. B 2001, 35, 789-813. [CrossRef]

6. Vansteenwegen, P.; van Oudheusden, D. Developing railway timetables which guarantee a better service. Eur. J. Oper. Res. 2006, 173, 337-350. [CrossRef]

7. Vansteenwegen, P.; van Oudheusden, D. Decreasing the passenger waiting time for an intercity rail network. Transp. Res. B 2007, 41, 478-492. [CrossRef]

8. Dariano, A.; Pacciarelli, D.; Pranzo, M. A branch and bound algorithm for scheduling trains in a railway network. Eur. J. Oper. Res. 2007, 183, 643-657. [CrossRef]

9. Dessouky, M.; Hall, R.; Nowroozi, A.; Mourikas, K. Bus dispatching at timed transfer transit stations using bus tracking technology. Transp. Res. C 1999, 7, 187-208. [CrossRef]

10. Assad, A.A. Modelling of rail networks: Toward a routing/makeup model. Transp. Res. B 1980, 14, 101-114. [CrossRef]

11. Li, Z.C.; Lam, W.H.; Wong, S.C.; Sumalee, A. Design of a rail transit line for profit maximization in a linear transportation corridor. Transp. Res. E 2012, 48, 50-70. [CrossRef]

12. Lee, Y.; Chen, C.Y. A heuristic for the train pathing and timetabling problem. Transp. Res. B 2009, 43, 837-851. [CrossRef]

13. Cao, Z.; Yuan, Z.; Zhang, S. Performance Analysis of Stop-Skipping Scheduling Plans in Rail Transit under Time-Dependent Demand. Int. J. Environ. Res. Public Health 2016, 13, 707. [CrossRef] [PubMed]

14. Zhou, X.; Zhong, M. Single-track train timetabling with guaranteed optimality: Branch-and-bound algorithms with enhanced lower bounds. Transp. Res. B 2007, 41, 320-341. [CrossRef]

15. Bertsimas, D.; Sim, M. The price of robustness. Oper. Res. 2004, 52, 35-53. [CrossRef]

16. Flamini, M.; Pacciarelli, D. Real time management of a metro rail terminus. Eur. J. Oper. Res. 2008, 189, 746-761. [CrossRef]

17. Meng, L.; Zhou, X. Robust single-track train dispatching model under a dynamic and stochastic environment: a scenario-based rolling horizon solution approach. Transp. Res. B 2011, 45, 1080-1102. [CrossRef]

18. Corman, F.; D'Ariano, A.; Pacciarelli, D. Bi-objective conflict detection and resolution in railway traffic management. Transp. Res. C 2012, 20, 79-94. [CrossRef]

19. Louwerse, I.; Huisman, D. Adjusting a railway timetable in case of partial or complete blockades. Eur. J. Oper. Res. 2014, 235, 583-593. [CrossRef]

20. Cacchiani, V.; Huisman, D.; Kidd, M.; Kroon, L.; Toth, P.; Veelenturf, L.; Wagenaar, J. An overview of recovery models and algorithms for real-time railway rescheduling. Transp. Res. B 2014, 63, 15-37. [CrossRef]

21. Nesheli, M.M.; Ceder, A.A. Improved reliability of public transportation using real-time transfer synchronization. Transp. Res. C 2015, 60, 525-539. [CrossRef]

22. Xu, X.; Li, K.; Yang, L.; Ye, J. Balanced train timetabling on a single-line railway with optimized velocity. Appl. Math. Model. 2014, 38, 894-909. [CrossRef] 
23. Vuchic, V.R. Urban Transit: Operation, Planning and Economics; John Wiley \& Sons: Hoboken, NJ, USA, 2005.

24. Jamili, A.; Aghaee, M.P. Robust stop-skipping patterns in urban railway operations under traffic alteration situation. Transp. Res. C 2015, 61, 63-74. [CrossRef]

25. Cao, Z.; Yuan, Z.; Li, D. Estimation method for a skip-stop operation strategy for urban rail transit in China. J. Mod. Transp. 2014, 22, 174-182. [CrossRef]

26. Sato, K.; Kei, T.; Norio, T. A MIP-based timetable rescheduling formulation and algorithm minimizing further inconvenience to passengers. J. Rail Trans. Plan. Manag. 2013, 3, 38-53. [CrossRef]

27. Gao, Y.; Kroon, L.; Schmidt, M.; Yang, L. Rescheduling a metro line in an over-crowded situation after disruptions. Transp. Res. B 2016, 93, 425-449. [CrossRef]

28. Cao, Z.; Yuan, Z.; Li, D. Synchronization and coordination optimization model of urban rail transit. J. Southeast Univ. 2016, 46, 221-225. (In Chinese)

29. Wang, Y.; Su, S.; Pan, X.; Cao, F.; Tang, T.; Ning, B.; De Schutter, B. Integrated line planning and train scheduling for an urban rail transit line. Transp. Res. Rec. 2016, 2540, 66-75. [CrossRef]

30. Wang, Y.; Tang, T.; Ning, B. Passenger-demands-oriented train scheduling for an urban rail transit network. Transp. Res. C 2015, 60, 1-23. [CrossRef]

31. Yan, Y.; Meng, Q.; Wang, S.; Guo, X. Robust optimization model of schedule design for a fixed bus route. Transp. Res. C 2012, 25, 113-121. [CrossRef]

32. Wirasinghe, S.C. Initial planning for urban transit systems. In Advanced Modeling for Transit Operationsand Service Planning; Lam, W.H.K., Bell, M.G.H., Eds.; Elsevier Science Ltd.: London, UK, 2003.

33. Furth, P.G.; Hemily, B.; Muller, T.H.J.; Strathman, J.G. TCRP Synthesis of Transit Practice 113. In Using Archived AVL-APC Data to Improve Transit Performance and Management; Transportation Research Board of the National Research Council: Washington, DC, USA, 2006.

(C) 2017 by the authors. Licensee MDPI, Basel, Switzerland. This article is an open access article distributed under the terms and conditions of the Creative Commons Attribution (CC BY) license (http:/ / creativecommons.org/licenses/by/4.0/). 